\title{
A DINÂMICA ENTRE OBJETIVOS, CONTROLE E ÉTICA: UM ESTUDO DE CASO SOBRE COMPLIANCE NO GERENCIAMENTO DE PROJETOS
}

Graziela Darla Araujo Galvão (graziela.galvao@usp.br) - Departamento de Engenharia de Produção, USP.

Marly Monteiro de Carvalho (malymc@usp.br) - Departamento de Engenharia de Produção, USP.

\section{RESUMO}

A literatura que reúne compliance e gerenciamento de projetos tende a concentrar-se em aspectos legais, como legislação trabalhista, do meio ambiente, anticorrupção, etc., oferecendo menor ênfase aos cumprimentos das normas, políticas e processos corporativos (regulamentação interna). Na prática, compliance com os processos e políticas organizacionais é considerada uma condição sine qua non para manter os negócios alinhados aos objetivos estratégicos e necessidades do negócio. Este cenário também alude uma área nebulosa na governança de projetos na prática, eventualmente expondo as organizações a eventuais falhas de compliance em relação às politicas e aos processos organizacionais. Este estudo tem como objetivo descrever os aspectos presentes na dinâmica entre a busca pelos resultados do projeto, o sistema de controles internos e a ética, por uma perspectiva de compliance em relação aos processos organizacionais. Pesquisa de natureza qualitativa, exploratória e descritiva, desenvolvida por meio de um estudo de caso único. Dados obtidos através de entrevistas realizadas com 10 gerentes de projetos quanto às práticas adotadas no desempenho de suas funções e sua aderência com os processos da metodologia de gestão de projetos existente. A organização mantém códigos explícitos de conduta e de valor estratégico, entretanto, dissonam da forma como constrói os seus mecanismos de controle e se posicionam em termos de objetivos de projetos, evidentemente centrados em uma perspectiva de resultado financeiro. A inconsistência, aparentemente no nível da cultura organizacional, poderia levar à descrença no código e baixa aderência aos processos e metodologia, como se observou no caso. Os achados desta pesquisa sugerem que não basta um forte código de ética, existência de metodologia, de processos e de um programa de capacitação em gerenciamento de projetos para que se alcance bons resultados em termos de compliance. É fundamental que haja uma coerência geral, com os valores corporativos transcritos em objetivos e critérios de sucesso nos projetos, além de mecanismos de controle adequados. Caso contrário, os gerentes de projetos terão comportamento igualmente inconsistente, podendo implicar em baixo nível de compliance com processos, métodos, valores e políticas organizacionais.

Palavras-chave: Gerenciamento de projeto; Compliance; Controles internos; ética

Área: Gestão de Projetos de Desenvolvimento de Produtos e Serviços

\section{INTRODUÇÃO}

O aumento consistente da importância dos projetos para as organizações tem o reconhecimento dos mais diversos setores econômicos, segmentos industriais e países (SVEJVIG; ANDERSEN, 2015). Entretanto, a base conceitual, modelos e metodologias de gestão de projetos utilizados parecem não ter acompanhado o mesmo ritmo evolutivo, 
mantendo-se centrados em uma perspectiva racionalista e tecnocrática, de certa forma destacados do contexto organizacional (SVEJVIG; ANDERSEN, 2015). A obtenção de metas de orçamento, prazo e requerimentos do projeto, direcionadores clássicos para a avaliação de desempenho e sucesso do projeto, já não seriam necessariamente autossuficientes para alcançar os objetivos de negócio e cumprimento dos múltiplos critérios e diretrizes que compõem a conjuntura corporativa (TOO; WEAVER, 2014).

A ponte entre a governança corporativa e o gerenciamento dos projetos é denominada de governança de projetos, a qual demanda limites que superam aqueles do próprio projeto também dito organização temporária - ao compreender a estrutura, dinâmica e mecanismos da organização que o abarca (organização permanente), bem como o entorno que adapta o ambiente de negócios (BIESENTHAL; WILDEN, 2014). Na mesma linha, Müller (2009) posiciona a governança de projetos no domínio que compreende o sistema de valores, as responsabilidades, os processos e as políticas que conduzem os projetos à conquista dos objetivos organizacionais, no melhor interesse de todas as partes interessadas, internas e externas, além da própria corporação. Nesta perspectiva, o projeto, mesmo sendo considerado uma organização temporária, continua sujeito ao compliance com as políticas, normas e procedimentos da organização permanente, além de quaisquer requisitos legais ou regulamentares aplicáveis (TOO; WEAVER, 2014)

Apesar da literatura que aborda simultaneamente governança e gerenciamento de projetos ainda colocar pouca ênfase em "como governar" melhor os projetos, o número de documentos relacionados indica que o tema surge como cada vez mais relevante (BIESENTHAL; WILDEN, 2014) Müller et al., 2014).

Em uma investigação na literatura compreendendo gerenciamento de projetos e compliance, percebeu-se que usualmente as pesquisas centram-se no atendimento à regulamentação externa à organização, como a lei norte-americana SOX, regulamentações ambientais, trabalhistas, etc., empregando menor ênfase às políticas e processos inerentes à própria organização, o que já sugere alguma aridez neste campo de estudo. Este cenário também alude uma área nebulosa na governança de projetos na prática, eventualmente expondo as organizações a eventuais falhas de compliance em relação às políticas e aos processos organizacionais.

Destas lacunas, emerge como objetivo deste trabalho, descrever os aspectos presentes na dinâmica entre a busca pelos resultados do projeto, o sistema de controles internos e a resposta ética dos participantes, por uma perspectiva de compliance em relação às políticas e aos processos organizacionais.

\section{REVISÃO DA TEORIA}

\subsection{O "Pano de Fundo": Governança corporativa e de projetos}

Em meados do século XX, Robert Monks lançou as bases do que se compreende hoje como governança corporativa, ao identificar que a legislação vigente à época, basicamente orientadas a coibir a formação de cartéis em processos de compras, não seria suficiente para proteger os acionistas e o mercado de eventuais práticas inadequadas por parte dos administradores. Monks propunha a necessidade de autocontrole das empresas, de maneira a atender não somente à legislação, mas de se manter transparência no fluxo de informações, além da prestação responsável de contas (ANDRADE; ROSSETTI, 2014).

A governança opera em níveis mais elevados da organização, podendo ser considerada como um conceito integrador, à medida que busca a conexão entre o micro e macro-nível da 
organização, inclusive entre os projetos e este nível estratégico corporativo (MÜLLER; PEMSEL; SHAO, 2014). A faixa de intersecção entre a governança corporativa e o gerenciamento dos projetos é denominada de governança de projetos, a qual compreende a estrutura, dinâmica e mecanismos da organização permanente, bem como o entorno que modela o ambiente de negócios (BIESENTHAL; WILDEN, 2014). Na mesma linha, Müller (2009) situa a governança de projetos no domínio que compreende o sistema de valores, as responsabilidades, os processos e as políticas que conduzem os projetos à consecução dos objetivos organizacionais, no melhor interesse de todas as partes interessadas, internas e externas, além da própria corporação que os abarca. Biesenthal \& Wilden (2014) e Müller et al. (2014) consideram a governança de projetos como um fator primordial para que se alcance o sucesso do projeto, trazendo valor de longo prazo para a organização, em compliance com as regras e políticas organizacionais.

\subsection{O Compliance em projetos}

A literatura sobre compliance concentra-se em grande número, nos aspectos externos à organização, especificamente acerca da regulamentação legal, como ambiental, da segurança e saúde ocupacional, de combate à corrupção, dos códigos da construção civil, etc. Entretanto, ao avaliar e estruturar as práticas de governança corporativa de uma empresa, os acionistas e gerentes devem considerar não apenas o cumprimento dos regulamentos, mas também os fatores organizacionais e ambientais em que a empresa opera, pois podem contribuir para a eficácia da governança corporativa, incluindo as políticas e processos (GHOFAR; ISLAM, 2015).

Especificamente compliance no domínio dos projetos, Too \& Weaver ( 2014) destacam que, mesmo sendo uma organização temporária, estes devem estar em compliance com toda a regulamentação externa a que a organização permanente está sujeita, bem como aos processos, políticas, objetivos e estratégia corporativos. Pinto \& Slevin (1988) apontam que um dos elementos cruciais para a implementação de sistemas de gerenciamento de projetos é a medição e controle das técnicas e táticas utilizadas, em conformidade com os critérios de sucesso adotados pela organização.

Neste sentido, a compliance com os processos - considerado um dos mais importantes ativos organizacionais - seria considerada uma condição sine qua non para manter o alinhamento e desempenho organizacionais (EL KHARBILI et al., 2008). Já Klein, Beranek, Martz \& Jiang (2006) constataram um efeito positivo no desempenho do projeto e da equipe do projeto, quando atua-se em acordo com o conjunto de processos organizacionais. Visão compartilhada por Kerzner (2011) e por Patah \& Carvalho (2013), os quais também relacionam o melhor desempenho dos projetos com o uso de processos, boas práticas ou metodologia adequados de gerenciamento de projetos. Müller (2009) destaca o risco de conflitos e inconsistências na aplicação dos processos e uso dos recursos, causando ineficiências dispendiosas que afetam negativamente a rentabilidade final do projeto.

\subsection{Controles internos}

Controles internos são os dispositivos da governança que visam trazer uma garantia razoável para o atingimento dos objetivos, de maneira correta e oportuna, em compliance com as regras em voga e com a mínima utilização de recursos (COSO, 2013). O desenvolvimento e escolha destes dispositivos estão sustentados na Teoria de Controle, a qual estuda os mecanismos pelos quais uma pessoa ou grupo em uma organização buscam garantir que outros atuem no sentido de alcançar objetivos organizacionais (KIRSCH, 1996).

Por uma perspectiva de formalidade e intensidade, o controle iria do tipo formal, ininterrupto e enérgico, até o autocontrole, informal e baseado em valores morais e éticos. Os controles 
formais afetam o controlado com base no seu desempenho em termos de resultados ou conformidade aos processos, incluindo dois tipos: (a) controle de comportamento (avaliam a forma como os procedimentos são seguidos); e (b) controle de resultados (avaliam o alcance das metas). Os controles informais influenciam os controlados por meio de estratégias sociais e da autogestão, com base em objetivos compartilhados, também incluindo dois tipos: (c) controles de clã (estabelecem valores compartilhados e metas de grupo); e (d) autocontrole (permitem ao indivíduo estabelecer os seus próprios objetivos de forma autônoma) (OUCHI, 1979) (KIRSCH; CUMMINGS, 1996) (LIU, 2015b).

Quanto à aplicabilidade, Etzioni (1989) relacionou o tipo de controle e de autoridade, com a natureza organizacional, sugerindo que organizações especializadas (orientadas a processos, com alto nível de especialização, mecanicista, autocráticas) demandariam controles mais formais e burocráticos, ao passo que aquelas não especializadas (produção mediante objetivos específicos e controles de metas, tipicamente orientadas a projetos, abordagem orgânica / adaptativa) teriam melhor aderência com os controles mais informais, brandos e orgânicos ((KIRSCH, 1997). Por outro lado, (LIU, 2015a) concluiu que apenas controles informais seriam pouco efetivos em projetos complexos e de alto risco.

A arte da boa governança, desta forma, seria a busca pelo equilíbrio adequado entre os processos restritivos para prevenir a má administração, porém ainda auxiliando a liberdade administrativa para apoiar o crescimento e a inovação efetivos, facultando a realização dos objetivos estratégicos da organização (TOO; WEAVER, 2014). Em outras palavras, o uso de controles adequados aos objetivos seria um fator principal para a sua eficácia (MÜLLER, 2009).

\section{4 Ética}

A ética pode ser definida como uma indagação sobre a natureza e os alicerces da moralidade e, esta última, significa aos julgamentos morais, aos padrões e às regras de conduta (THONG; YAP, 1998). Segundo Hartman, Desjardins \& McDonald (2008), a ética diz respeito a como o ser humano age, decide e se comporta na prática ou, por uma perspectiva aristotélica, o conjunto de virtudes que explicam e prescrevem as ações dos indivíduos, complementam os autores.

As pesquisas sobre ética em projetos também transcorrem segundo diversas abordagens construídas sobre perspectivas externas ao projeto (responsabilidade socioambiental, direitos de propriedade, segurança ocupacional, etc.), ou internas, de como se comporta o profissional do projeto. Sendo esta última, a de interesse ao escopo do presente trabalho, com particular atenção à forma como o profissional decide em relação ao compliance com os processos corporativos no gerenciamento de projetos.

Em relação à formação profissional, a concentração de atenção no conhecimento formal, padronizado e universal, levaria ao detrimento da legitimidade de outras formas de conhecimento, como criatividade, autonomia e discernimento, além das questões éticas e morais dos praticantes (HODGSON; CICMIL, 2007).

Do ponto de vista de avaliação do projeto, existiriam tensões éticas oriundas da formação do profissional com base em padrões e modelos de avaliação das competências provenientes de diferentes escolas, além de distintas abordagens de atributo e de desempenho (BREDILLET; TYWONIAK; DWIVEDULA, 2015). O conjunto de valores ponderaria a avaliação de competências em outras abordagens. Assim, o melhor julgamento dos resultados de um projeto dependeria da moderação das partes interessadas, que poderia encontrar soluções mais éticas aos dilemas na análise de projetos (DE BRUCKER; MACHARIS; VERBEKE, 2013). Segundo Etzioni (1989), valores morais e éticos podem orientar o comportamento dos 
indivíduos, portanto, também atuando como forma de controle, especificamente, embasando o autocontrole.

\section{ABORDAGEM METODOLÓGICA}

Este trabalho tem natureza qualitativa, exploratória e descritiva de um fenômeno contemporâneo no contexto social, tendo por estratégia o estudo de caso (YIN, 2015). O estudo de caso foi realizado na unidade brasileira de uma multinacional de grande porte, de origem europeia, atuante no segmento de projetos de automação industrial, a qual corresponde à unidade de análise da presente pesquisa.

O roteiro semiestruturado de entrevista contemplou questões sustentadas pelo referencial teórico do presente estudo, incluindo: (i) a percepção dos entrevistados quanto à metodologia e processos de gerenciamento de projetos adotados pela companhia, sobre as ferramentas disponíveis e programas de capacitação para gerentes de projetos; (ii) a percepção dos entrevistados quanto às políticas de risco e valores estratégicos; (iii) a forma como os entrevistados gerenciavam os projetos, ou seja, os processos seguidos, técnicas e ferramentas utilizadas, as políticas seguidas, e as formas como resolviam os problemas relatados.

Os entrevistados foram selecionados pelo Especialista de Operações Sênior, os quais, segundo este, ofereceriam a melhor representatividade considerando experiência, formação, desempenho e gênero. A caracterização da amostra entrevista está disposta na figura 1.

Tabela 1. Perfil dos Entrevistados

\begin{tabular}{|c|c|c|c|c|}
\hline \multicolumn{7}{|c|}{ PERFIL DOS ENTREVISTADOS } \\
\hline Entrevistado & Gênero & Função & Tempo na Função & Área Anterior \\
\hline GP01 & Masculino & Gerente de Projeto & 7,5 anos & Vendas \\
\hline GP02 & Masculino & Gerente de Projeto & 2,5 anos & Vendas \\
\hline GP03 & Masculino & Gerente de Projeto & 3,5 anos & Engenharia \\
\hline GP04 & Feminino & Gerente de Projeto & 4,0 anos* & Engenharia \\
\hline GP05 & Masculino & Gerente de Projeto & 5,0 anos & Engenharia \\
\hline GP06 & Masculino & Gerente de Projeto & 3,0 anos & Projetos \\
\hline GP07 & Masculino & Gerente de Projeto & 3,0 anos & Engenharia \\
\hline GP08 & Masculino & Gerente de Projeto & 2,0 anos & Engenharia \\
\hline GP09 & Feminino & Administradora de Contratos* & 5,0 anos & Vendas \\
\hline GP10 & Masculino & Gerente de Projeto & 1,0 ano & Engenharia \\
\hline
\end{tabular} * Há 19 anos trabalhando em projetos na organização, mas há 4 na posição.
** Gerencia pequenos projetos, sem envolvimento de engenharia.

A análise contempla as seguintes proposições:

- (P1) Os gerentes de projetos atuam em compliance com a metodologia, políticas, procedimentos e processos organizacionais;

- (P2) A organização dispõe de mecanismos de controles internos coerentes com os critérios formais e tácitos de sucesso do projetos e efetivos;

- (P3) A organização investe em programas para compliance com o código de ética corporativa.

\section{ANÁLISE E DISCUSSÃO DOS RESULTADOS}

\subsection{O compliance com os processos, políticas e metodologia internos}

O primeiro passo para se verificar o compliance com processos, políticas e metodologias foi identifica-los, assim como os treinamentos conferidos aos gerentes de projetos. 
A metodologia de gerenciamento de projetos é abrangente, madura e global, baseada nos guias de boas práticas. Há um amplo e robusto programa de formação de gerente de projetos, com diversos estágios para obtenção de certificações com profundidade distintas. Quanto maior for o nível alcançado pelo gerente de projetos, maior será o seu salário.

Apesar da aprovação generalizada aos cursos e de evidências documentais da existência de uma metodologia de gestão de projetos, curiosamente os entrevistados por vezes não a reconhecem ou não a aprovam: "não sigo muito, pois acabo me perdendo no caminho" (GP1); "sinto muita falta de metodologia, como tive em outras empresas" (GP2); "cada um faz do seu jeito, muito embora acredite que a da empresa seja muito boa" (GP4); "Acho que não é seguida. Cada um faz do seu jeito. Não existe padrão, ou não é usado" (GP8).

Em relação aos usos das ferramentas e seguimento dos processos indicados na metodologia e oferecidos nos treinamentos é insipiente, conforme se observa na tabela 2. Novamente, os depoimentos reconhecem a existência do ferramental, mas não o aplicam: "Existem muitas ferramentas, mas o dinamismo (tempo), não permite que se use" (GP8); “(...) um módulo que eu fiz no mês passado, aplicando todas as ferramentas... bem prático e dinâmico que fez as pessoas aprenderem a usar as ferramentas" (GP10). Nas entrevistas, muitos declararam não usar ferramentas básicas, que fazem parte da metodologia, como cronograma, WBS, matriz de responsabilidades, etc. Há uma total variedade de formas de atuação, sem qualquer evidência de uniformidade.

Tabela 2. Sumarizando o Compliance com a Metodologia de Projetos

\begin{tabular}{|c|c|c|c|c|c|c|c|c|c|c|}
\hline Argumento & $\mathrm{GP}_{1}$ & $\mathrm{GP}_{2}$ & $\mathrm{GP}_{3}$ & $\mathrm{GP}_{4}$ & $\mathrm{GP}_{5}$ & $\mathrm{GP}_{6}$ & $\mathrm{GP}_{7}$ & $\mathrm{GP}_{8}$ & $\mathrm{GP}_{9}$ & $\mathrm{GP}_{10}$ \\
\hline $\begin{array}{l}\text { Utiliza ferramentas de } \\
\text { controle de escopo, } \\
\text { conforme a metodologia e } \\
\text { treinamentos recebidos }\end{array}$ & Q & Q & Q & Q & Q & Q & Q & Q & प & Q \\
\hline $\begin{array}{l}\text { Utiliza ferramentas de } \\
\text { controle de custo, } \\
\text { conforme a metodologia e } \\
\text { treinamentos recebidos }\end{array}$ & Q & Q & 口 & प & प & Q & Q & Q & Q & Q \\
\hline $\begin{array}{l}\text { Utiliza ferramentas de } \\
\text { controle de prazo, } \\
\text { conforme a metodologia e } \\
\text { treinamentos recebidos }\end{array}$ & Q & Q & Q & Q & Q & Q & Q & Q & प & Q \\
\hline $\begin{array}{l}\text { Utiliza ferramentas de } \\
\text { controle de risco, } \\
\text { conforme a metodologia e } \\
\text { treinamentos recebidos }\end{array}$ & Q & Q & 口 & प & प & Q & प & Q & प & Q \\
\hline $\begin{array}{l}\text { Utiliza a matriz de } \\
\text { responsabilidades, } \\
\text { conforme a metodologia e } \\
\text { treinamentos recebidos }\end{array}$ & Q & Q & Q & Q & Q & Q & Q & Q & Q & Q \\
\hline $\begin{array}{l}\text { Registra as lições } \\
\text { aprendidas no projeto, } \\
\text { conforme a metodologia e } \\
\text { treinamentos recebidos }\end{array}$ & Q & Q & Q & प & Q & प & Q & प & 口 & 口 \\
\hline $\begin{array}{l}\text { Não utiliza controles } \\
\text { paralelos aos estipulados } \\
\text { pela metodologia }\end{array}$ & Q & 口 & Q & Q & Q & Q & Q & Q & Q & Q \\
\hline
\end{tabular}

- Argumento conforme $\bigcirc$ Argumento parcialmente conforme $\quad \times$ Argumento não conforme

Quanto a política de riscos, à medida que existe uma ferramenta de riscos em projetos, que já está alinhada à política. É dever dos gerentes de projetos, não só a preencherem corretamente, mas monitorar os riscos ao longo da vida do projeto. Entretanto, somente dois dos entrevistados estavam em compliance com a política (GP1 e GP3). Outros dois, não cumprem todos os quesitos (GP6 e GP8), o que do ponto de vista do compliance significaria "não conforme". Os demais apenas preenchem mecanicamente a ferramenta, sem preocupação com a apuração das informações e apenas no início do projeto, para atenderem a eventuais auditorias. 
Desta forma, seja por uma perspectiva de processos, metodologia ou políticas, a proposição P1 é refutada nesta pesquisa.

\subsection{Os mecanismos de controle no gerenciamento de projetos}

Ao se avaliar a forma como a organização controla os projetos, percebeu-se que há uma reunião mensal com cada gerente de projetos coordenada pela controladoria de projetos, para revisão geral dos avanços e problemas no período. Segundo o procedimento, a reunião deveria contemplar os progressos (escopo), o desempenho de prazo, o desempenho financeiro, a revisão dos riscos e oportunidades e os indicadores de qualidade, incluindo a satisfação do cliente (valor estratégico). Entretanto, mediante os depoimentos de todos entrevistados, as reuniões restringem-se ao desempenho financeiro: "tem foco financeiro" (GP1); "o tema é financeiro. Eu levo problemas, mas o assunto relevante é o orçamento do projeto" (GP4).

Considerando o depoimento de todos os entrevistados, identificou-se dois tipos de controle da empresa: (i) formal e de resultados, especificamente em metas financeiras; e (ii) autocontrole (informal) (OUCHI, 1979) (KIRSCH; CUMMINGS, 1996) (LIU, 2015b).

Considerando-se que os valores corporativos exaltam muitos outros aspectos além dos financeiros, não contemplados nos mecanismos de controles identificados, a proposição P2 é refutada.

\subsection{A ética no gerenciamento dos projetos}

O estudo também investigou o posicionamento da organização em relação à ética nos negócios. Foi constatado que a empresa mantém e divulga efetivamente um código de conduta global, aplicável a todos colaboradores, administradores e membros do conselho, bem como stakeholders (incluindo relações com fornecedores, clientes e governos). A organização declara e divulga os seus valores éticos, os quais explicitamente afirmam que o desempenho não deve ser avaliado em função dos resultados, mas inclusive a forma como o resultado se construiu. A subsidiária brasileira, inclusive, alcançou e superou as condições mínimas para receber uma certificação brasileira conferido às empresas éticas. Os entrevistados demonstraram o conhecimento geral sobre o código de conduta ética. Desta forma, a organização que investe efetivamente em um código de conduta ética que, entre outras coisas, determina o compliance com as políticas corporativas.

Entretanto, quando inquirido quanto ao desempenho financeiro dos projetos, o entrevistado GP8 relatou que faz manobras financeiras indevidas, transferindo custos de projetos "muito carregados", para outros com margem disponível. Reiterou ainda, que esta seria uma prática comum entre os gerentes de projetos. A prestação responsável de contas é uma das bases da governança corporativa (ANDRADE; ROSSETTI, 2014).

\section{CONCLUSÃO}

Hirschi (1969) argumenta que a obediência dos indivíduos aos códigos depende da sua crença moral nestes. A organização mantém códigos explícitos de conduta e de valor estratégico, entretanto, dissonam da forma como constrói os seus mecanismos de controle e se posicionam em termos de objetivos de projetos, evidentemente centrados em uma perspectiva de resultado financeiro. A inconsistência, aparentemente no nível da cultura organizacional, poderia levar à descrença no código e baixa aderência aos processos e metodologia, como se observou no caso.

Os achados desta pesquisa sugerem que não basta um forte código de ética, existência de metodologia, de processos e exemplar programa de capacitação em gerenciamento de projetos. É fundamental que haja uma coerência holística. Se os valores corporativos não 
estiverem devidamente transcritos em objetivos e critérios de sucesso nos projetos, e balizados por mecanismos de controle adequados, o comportamento igualmente inconsistente dos gerentes de projetos parece fazer sentido. A aparente confusão observada no caso estudado, parece causar o baixo compliance com as políticas, metodologia e processos corporativos.

\section{REFERÊNCIAS}

ANDRADE, A.; ROSSETTI, J. P. Governança corporativa: fundamentos, desenvolvimento e tendências. 7. ed. São Paulo: Editora Atlas, 2014.

BIESENTHAL, C.; WILDEN, R. Multi-level project governance: Trends and opportunities. International Journal of Project Management, v. 32, n. 8, p. 1291-1308, 2014.

BREDILLET, C.; TYWONIAK, S.; DWIVEDULA, R. What is a good project manager? An Aristotelian perspective. International Journal of Project Management, v. 33, n. 2, p. 254-266, 2015.

COSO. Internal control - integrated framework: executive summary. [s.1.] Committee of Sponsoring Organizations of the Treadway Commission, 2013.

DE BRUCKER, K.; MACHARIS, C.; VERBEKE, A. Multi-criteria analysis and the resolution of sustainable development dilemmas: A stakeholder management approach. European Journal of Operational Research, v. 224, n. 1, p. 122-131, 2013.

EL KHARBILI, M. et al. Towards a framework for semantic business process compliance management. Proceedings of GRCIS, v. 2008, 2008.

ETZIONI, A. Organizações modernas. 8a. ed. São Paulo: Pioneira, 1989.

GHOFAR, A.; ISLAM, S. M. N. Corporate Governance and Contingency Theory. [s.l: s.n.].

HARTMAN, L.; DESJARDINS, J.; MACDONALD, C. Business ethics: decision making for personal integrity \& social responsibility. 3. ed. New York: McGraw-Hill, 2008.

HIRSCHI, T. Causes of delinquency. Berkeley: University of California Press, 1969. v. 47

HODGSON, D.; CICMIL, S. The politics of standards in modern management: making "the project"a reality. Journal of Management Studies, v. 44, n. 3, p. 431-450, 2007.

KERZNER, H. Gerenciamento de projetos: uma abordagem sistêmica para planejamento, programação e controle. Tradução d ed. São Paulo: Editora Edgard Blucher Ltda., 2011.

KIRSCH, L. J. The Management of Complex Tasks in Organizations: Controlling the Systems Development Process. Organization Science, v. 7, n. 1, p. 1-21, 1996.

KIRSCH, L. J. Portfolios of Control Modes and IS Project Management. Information Systems Research, v. 8, n. 3, p. 215-239, 1997.

KIRSCH, L. J.; CUMMINGS, L. L. Contextual influences on self-control of is professionals engaged in systems development. Accounting, Management and Information Technologies, v. 6, n. 3, p. 191-219, 1996.

KLEIN, G. et al. the Relationship of Control and Learning To Project Performance. Cybernetics and Systems, v. 37, n. 2-3, p. 137-150, 2006.

LIU, S. Effects of control on the performance of information systems projects: The moderating role of complexity risk. Journal of Operations Management, v. 36, p. 46-62, 2015a. 
LIU, S. How team risk and planning and control risk moderate the effects of clan and self control on the process performance of IT projects: the perspective of user liaisons. Information Development, v. 31, n. 1, p. 27-39, jan. 2015 b.

MÜLLER, R. Project governance. Farnham, Surrey, England ; Burlington, VT: Gower, 2009.

MÜLLER, R.; PEMSEL, S.; SHAO, J. Organizational enablers for governance and governmentality of projects: A literature review. International Journal of Project Management, v. 32, n. 8, p. 1309-1320, 2014.

OUCHI, W. G. A conceptual framework for the design of organizational control mechanisms. In: Readings in Accounting for Management Control. [s.1.] Springer, 1979. p. 63-82.

PATAH, L. A.; CARVALHO, M. M. DE. Sucesso a partir de investimento em metodologias de gestão de projetos. Production, v. 0, n. ahead, p. 00-00, 2013.

PINTO, J. K.; SLEVIN, D. P. Critical success factors in effective project implementation. Project management handbook, v. 479, 1988.

SVEJVIG, P.; ANDERSEN, P. Rethinking project management: A structured literature review with a critical look at the brave new world. International Journal of Project Management, v. 33, n. 2, p. 278-290, 2015.

THONG, J. Y. L.; YAP, C.-S. Testing an ethical decision-making theory: the case of softlifting. Journal of Management Information Systems, v. 15, n. 1, p. 213-237, 1998.

TOO, E. G.; WEAVER, P. The management of project management: A conceptual framework for project governance. International Journal of Project Management, v. 32, n. 8, p. 1382-1394, 2014.

YIN, R. K. Case study research design and methods. 5. ed. California: Sage Inc., 2015. 